\title{
APROXIMACIONES A LA ENSEÑANZA DEL ESPAÑOL COMO LENGUA DE HERENCIA
}

\author{
Marta Fairclough
}

\section{(9) $\odot \Theta \Theta$}

Esta obra está bajo una licencia Creative Commons

Reconocimiento-No Comercial-Sin Obra Derivada 



\title{
APROXIMACIONES A LA ENSEÑANZA DEL ESPAÑOL COMO LENGUA DE HERENCIA
}

\author{
APPROACHES TO TEACHING SPANISH AS A HERITAGE \\ LANGUAGE
}

\author{
Marta Fairclough
}

\begin{abstract}
RESUMEN
El objetivo de este artículo es ofrecer una visión general de las prácticas pedagógicas actuales en la enseñanza del español como lengua heredada en Estados Unidos, incluyendo algunas de las prácticas más innovadoras del campo. Después de una breve introducción a la situación socio-demográfica y lingüística de la lengua española en dicho contexto, se describen algunas de las principales características que definen al estudiantado de lengua de herencia y se mencionan los modelos pedagógicos vigentes en los programas de herencia y su presencia en las instituciones terciarias. La segunda parte se enfoca en ciertas prácticas pedagógicas innovadoras en la enseñanza del español como lengua de herencia. Dichas innovaciones se basan en una pedagogía crítica de la lengua (Fairclough, 1995) cuyo propósito es fomentar la concientización y el pensamiento crítico por medio de la incorporación de contenido de orden sociolingüístico en el aula, la adquisición multidialectal, el translenguar, la multialfabetización cultural (New London Group, 1996) y la interacción con la comunidad. Las prácticas innovadoras conducen a forjar una conexión más estrecha entre el estudiante de lengua de herencia y la comunidad de habla, que a su vez conlleva el mantenimiento de la lengua minoritaria en una situación de contacto lingüístico.

Palabras clave: bilingüismo, lengua de herencia, español en Estados Unidos, enseñanza del español, pedagogía.
\end{abstract}

\begin{abstract}
The purpose of this article is to offer a general view of current pedagogical practices in the teaching of Spanish as a heritage language in the United States, including some of the most innovative practices in the field. After a brief introduction to the socio-demographic and linguistic situation of the Spanish language in that context, the article describes some of the main characteristics of the heritage language learner and summarizes existing pedagogical models and their presence in tertiary institutions. The second part focuses on certain innovative pedagogical practices in the teaching of Spanish as a heritage language. Those innovations are based on the critical language pedagogy model (Fairclough, 1995), whose purpose is to promote language awareness y critical thinking by incorporating into the classroom sociolinguistic content, the acquisition of multiple dialects, translanguaging, cultural 'multiliteracies' (New London Group, 1996), and interaction with the community. These innovative practices lead to a stronger connection between the heritage language learner and the speech community, which contributes to the maintenance of the minority language in a linguistic contact situation.

Key words: bilingualism, heritage language, Spanish in the United States, teaching Spanish, pedagogy.
\end{abstract}

Dra. Marta Fairclough. Universidad de Houston. Profesora de Lingüística española. Directora del Spanish Heritage Language Program (SHL). Estados Unidos.

Correo electrónico: mfairclough@uh.edu

Recepción: 27- 05- 2015

Aceptación: 30- 08- 15 


\section{Introducción}

El objetivo de este artículo es ofrecer una visión general de las prácticas pedagógicas actuales en la enseñanza del español como lengua heredada (Spanish as a heritage language) en Estados Unidos, incluyendo algunas de las ideas más innovadoras dentro del campo y partiendo de perspectivas sociolingüísticas, psicolingüísticas y educativas. Después de una breve introducción a la situación socio-demográfica y lingüística del español en Estados Unidos, en la cual se incorporan algunas de las principales características que definen al estudiantado de lengua de herencia (y lo diferencian de los estudiantes de español como segunda lengua), se describen los modelos pedagógicos vigentes y su presencia en las instituciones terciarias (Beaudrie, 2012, 2015).

La segunda parte del artículo se enfoca en ciertas prácticas pedagógicas innovadoras en la enseñanza del español como lengua de herencia. Dichas innovaciones se basan en la importancia de incorporar contenido de orden sociolingüístico en la enseñanza, con un énfasis especial en la variación lingüística, para concientizar a los estudiantes sobre los aspectos sociopolíticos e ideológicos de la lengua (Freire, 1970) y darles la posibilidad de tomar sus propias decisiones linguiísticas (Leeman, 2014). A esta pedagogía crítica sobre la lengua (Fairclough, 1995) se suma el modelo de "multialfabetización" cultural (multiliteracies) (New London Group, 1996) cuyo propósito es promover el pensamiento crítico a través de la incorporación de otros tipos de discurso, como el arte, los blogs o los anuncios publicitarios (Parra, 2013; Samaniego y Warner, s.f.). Las mencionadas aproximaciones pedagógicas contribuyen a forjar una conexión entre estudiante de lengua de herencia y la comunidad de habla por medio de proyectos culturales, pasantías y estudios en otros países de habla hispana. Dichas conexiones se van expandiendo paulatinamente de la comunidad local hacia la global y conducen a un compromiso social cuyo objetivo primordial es promover el mantenimiento de la lengua de herencia en situaciones de contacto lingüístico.

\section{Contexto socio-demográfico y situación lingüística}

\subsection{El español como lengua de herencia en Estados Unidos}

En Estados Unidos la denominación de lengua de herencia se usa para nombrar las lenguas de las minorías entre las que no se incluye el inglés. En otros países, se prefieren términos como lengua (de la) comunidad, ancestral, étnica, inmigrante, nativa, original, materna, no oficial, minoritaria, regional, etc. La siguiente tabla presenta la distribución de las lenguas minoritarias más habladas en dicho país según datos del US Census Bureau (Ryan, 2013).

Tabla 1. La distribución de hablantes de las lenguas minoritarias en Estados Unidos

\begin{tabular}{|l|r|r|}
\hline Idioma & Número de hablantes & Porcentaje \\
\hline Español & 37579787 & $62 \%$ \\
\hline Chino & 2882497 & $4.8 \%$ \\
\hline Francés & 2055433 & $3.3 \%$ \\
\hline Tagalo & 1594413 & $2.6 \%$ \\
\hline Vietnamita & 1419539 & $2.3 \%$ \\
\hline
\end{tabular}




\begin{tabular}{|l|r|r|}
\hline Koreano & 1141227 & $1.9 \%$ \\
\hline Alemán & 1083637 & $1.8 \%$ \\
\hline Árabe & 951699 & $1.6 \%$ \\
\hline Ruso & 905843 & $1.5 \%$ \\
\hline Italiano & 723632 & $1.1 \%$ \\
\hline Portugués & 673566 & $1.1 \%$ \\
\hline Hindi & 648983 & $1.1 \%$ \\
\hline Polaco & 607403 & $1.0 \%$ \\
\hline Otros & 8309361 & $14 \%$ \\
\hline Total & $\mathbf{6 0 5 7 7 0 2 0}$ & $\mathbf{1 0 0 \%}$ \\
\hline
\end{tabular}

Más de sesenta millones de personas hablan un idioma que no es inglés en el hogar. De esta cifra, casi treinta y ocho millones son hablantes de español. Como se desprende de los datos de la Tabla 1, los hispanohablantes representan más de un $60 \%$ del total de lenguas minoritas en Estados Unidos. En la siguiente tabla se puede observar el aumento gradual de la población hispana desde el año 1980 hasta el último censo.

Tabla 2. Población hispana en Estados Unidos de 1980 a 2010 (Fuente: US Census Bureau, 2012)

\begin{tabular}{|c|c|c|r|}
\hline Año & Población total & $\begin{array}{c}\text { Población } \\
\text { hispana }\end{array}$ & $\begin{array}{c}\text { Porcentaje de } \\
\text { aumento }\end{array}$ \\
\hline 1980 & 226500000 & 14600000 & $6.4 \%$ \\
\hline 1990 & 248709803 & 22354059 & $9.0 \%$ \\
\hline 2000 & 281421906 & 37400000 & $13.3 \%$ \\
\hline 2010 & 308745000 & 50500000 & $16.3 \%$ \\
\hline
\end{tabular}

La Figura 1 ilustra la concentración de la población hispana en el sudoeste del país, donde viven más de tres cuartas partes de los hispanos. De acuerdo al censo nacional del 2010, los estados que cuentan con el mayor porcentaje de residentes hispanos son California (28\%) y Texas (19\%).

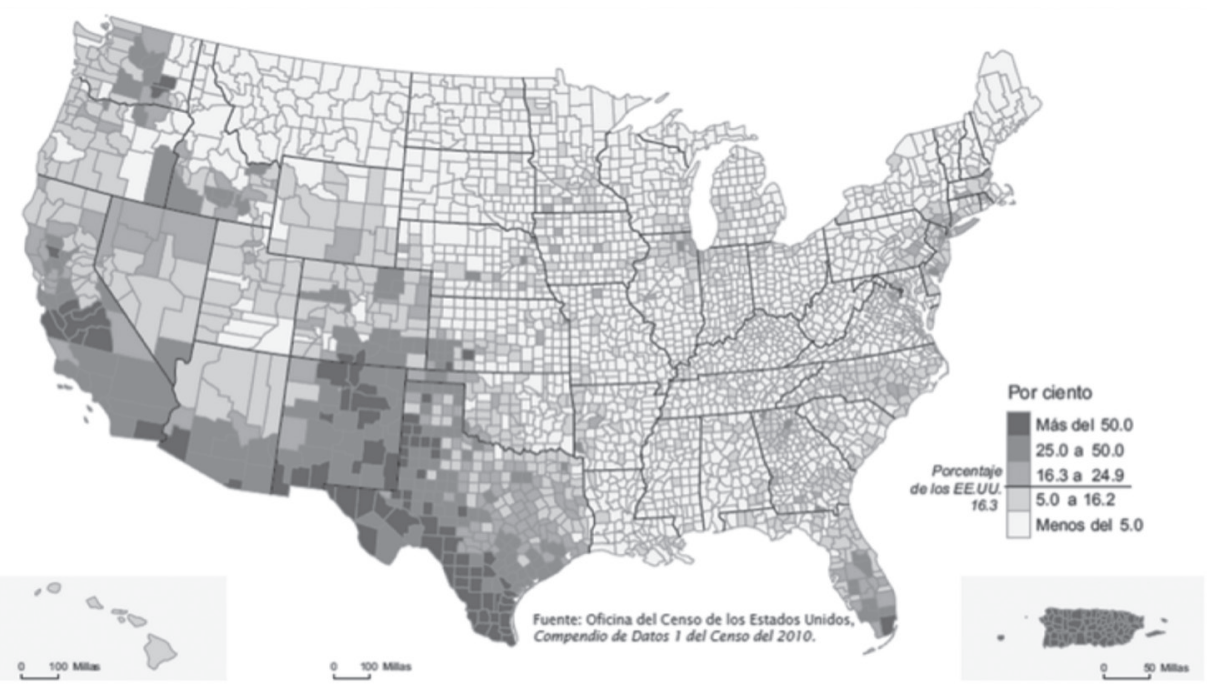

Figura 1. Distribución de la población hispana en Estados Unidos 
Los siguientes puntos presentan datos adicionales sobre los hispanos en Estados Unidos y su dominio de la lengua española según datos del censo y del Pew Hispanic Center (2013):

- Se proyecta que para el año 2050, la población hispana en los EE. UU. se triplique, alcanzando 132.8 millones de residentes.

- Aunque vienen de todos los países de habla hispana, casi 2/3 de los hispanos en Estados Unidos son de procedencia mexicana (65\%).

- Datos del 2013 indican que cerca del 66\% de la población hispana nació en Estados Unidos.

- Aproximadamente el $82 \%$ de los hispanos adultos puede conversar en español.

- El 38\% considera el español como lengua dominante y en el 24\% el inglés es la lengua preponderante; el restante $38 \%$ se considera bilingüe.

Varios de estos factores, incluyendo el considerable aumento de hispanos y el alto porcentaje de los nacidos en Estados Unidos conducen a un mayor interés en el estudio del español como lengua heredada, tanto como meta personal o profesional, sobre todo a niveles terciarios.

\subsection{El estudiante de español como lengua de herencia}

El estudiante de español como lengua de herencia (EELH) es aquel individuo proveniente de un hogar en donde se habla español, que además habla o solamente entiende la lengua de herencia, y que es hasta cierto punto bilingüe en inglés y español (definición basada en Valdés, 2005). Las principales características que definen el perfil lingüístico de los EELH incluyen las siguientes:

- $\quad$ proceden de diversos orígenes económicos, sociales, geográficos y lingüísticos;

- $\quad$ poseen un lazo afectivo con su lengua materna;

- aprenden la lengua de herencia en el ámbito familiar;

- en su mayoría, aprenden la lengua heredada (LH) oralmente, con escasa o nula exposición a las formas escritas;

- tienden a estar más familiarizados con la variedad coloquial que con el registro formal.

En la producción lingüística de los EELH suele notarse la presencia de simplificación morfosintáctica (p. ej., Lipski, 1993; Silva-Corvalán, 1994), un léxico limitado (p. ej., Valdés y Geoffrion-Vinci, 1998), cambio de código (p. ej., Carvalho, 2012; Fairclough, 2003), formas dialectales (p. ej., Bernal-Enríquez y Hernández-Chávez, 2003), transferencia (p. ej., SilvaCorvalán, 1994; Zentella, 1997) y lagunas lingüísticas (p. ej., Parodi, 2008).

Si bien los EELH constituyen un grupo heterogéneo, para fines pedagógicos se distancian enormemente de los estudiantes de español como segunda lengua (EESL), dados los lazos afectivos y socio-culturales que los unen a la lengua heredada, pero también por las marcadas diferencias lingüísticas entre los dos grupos.

\subsection{Principales diferencias entre el estudiante de EELH y el EESL}

Las principales diferencias lingüísticas entre los EELH y los EESL incluyen cierto conocimiento previo de la lengua aun en los niveles más bajos de habilidad lingüística de los EELH (p. ej., el reconocimiento léxico (Fairclough, 2011)). Además estos estudiantes suelen poseer una fonología más cercana a la de un hablante nativo que los EESL (Au, Knightly, Jun, 
y Oh, 2002; Oh y Au, 2005), diferente ritmo en el desarrollo gramatical en el salón de clase, diferentes contextos en los que tienden a cometer errores (oral versus escrito) y diferentes tipos de conocimiento al que recurren cuando se les asigna una tarea (intuitivo/implícito versus conocimiento metalingüístico) (Beaudrie, 2009; Bowles, 2011; Correa, 2011; Fairclough, 2005; Hislope, 2005; Mikulski, 2010; Montrul, 2011; Montrul y Bowles, 2010; Montrul, Foote, y Perpiñán, 2008; Potowski, Jegerski, y Morgan-Short, 2009).

Aun dejando de lado los factores socio-afectivos, tales como la motivación para aprender la LE o la identidad con el grupo minoritario al que pertenecen, dadas las numerosas disimilitudes de tipo lingüístico y de aprendizaje entre los dos grupos, es fundamental utilizar metodologías y materiales especialmente diseñados para los EELH para que el beneficio sea óptimo.

\subsection{Los modelos pedagógicos vigentes en los programas de herencia y su presencia en las instituciones terciarias}

El nivel de dominio en español disminuye marcadamente de una generación a la siguiente (Fishman, 2001; Veltman, 1983, 2000). Con el fin de promover el mantenimiento y revitalización de la lengua de herencia un número cada vez mayor de universidades ofrece cursos especializados para esta población estudiantil. De acuerdo con Beaudrie (2012), 40\% de las universidades estadounidenses con un mínimo del 5\% de estudiantes hispanos ofrece cursos de ELH: 26 estados y Washington D.C. La investigadora indica que la mayoría de las instituciones (81\%) ofrece uno o dos cursos; el resto, tres o más. Sin embargo, un gran número continúa ofreciendo exclusivamente los cursos tradicionales de español como segunda lengua o como lengua extranjera (ESL) en los que los EELH y EESL conforman las denominadas "clases mixtas" (Carreira, 2012).

En los cursos diseñados especialmente para los EELH, la modalidad estándar de la lengua española ha sido el foco de la enseñanza a través de los años (p. ej. Hidalgo, 1990, 1997; Valdés, 1978, 1997). Sin embargo, recientemente se ha tratado de enfatizar las diversidad lingüística y la concientización dialectal (Leeman, 2005; Martínez, 2003). En un estudio llevado a cabo por Beaudrie (2015), la investigadora analiza las metas de los cursos de ELH en más de 60 universidades públicas y privadas ubicadas en 15 estados diferentes de Estados Unidos. El análisis de los datos se concentra en los enfoques hacia la variación lingüística en dichas instituciones, que van de la erradicación de la variedad que el estudiante trae consigo al salón de clase (ya prácticamente en desuso) a aproximaciones que fomentan la expansión y la apreciación de la lengua materna (los más utilizados), el uso según el contexto (más o menos formal) y finalmente a modelos críticos, que además de enseñar el dialecto estándar, intentan validar la variedad hablada por los estudiantes por medio de la concientización del alumnado en cuanto a los aspectos sociopolíticos e ideológicos de la lengua.

\section{La enseñanza del español como LH: marco teórico y prácticas pedagógicas}

La mayoría de programas de LH tiene como objetivo enseñar el dialecto estándar y fundar respeto por las variedades del hogar (p. ej., Jo, 2001; Kondo-Brown, 2010; Rouchdy, 2002; Valdés, 2005, 2006, entre muchos otros); no obstante, cada vez más investigaciones apuntan hacia la necesidad de enseñar dialectos alternativos para expandir el repertorio lingüístico de los EELH mediante (a) la adquisición del conocimiento lingüístico 
pertinente para promover el entendimiento de la conexión entre lenguaje y poder por parte de los estudiantes; (b) el desarrollo de una conciencia lingüística a través de técnicas contrastivas para identificar y adquirir formas lingüísticas adicionales; (c) la promoción de la multialfabetización, que contribuye a la competencia translingüística y transcultural; y (d) la interacción con la comunidad.

Cada vez más programas parecen estar reconociendo el valor de incluir las variedades del hogar en las aulas, entendidas no solo como un punto de partida para enseñar la variedad estándar, sino como herramientas prácticas de enseñanza. Gutiérrez y Fairclough (2006) proponen que además de enseñar "la forma híbrida y simplificada de la lengua" que domina la mayoría de programas de lenguas, "la instrucción debería evolucionar paulatinamente de una concientización de la variación lingüística a un uso de dialectos alternativos y de un enfoque en variedades, registros y estilos locales a otras variedades, registros y estilos del español alrededor del mundo" (184, mi traducción, énfasis mío). La adquisición de múltiples variedades lingüísticas dota al estudiante de más opciones comunicativas y fomenta un modelo multidialectal (Fairclough, s.f.). Aunque es muy probable que en muchas situaciones el dialecto estándar sea necesario, también habrá otros contextos en los que las variedades del hogar sean indispensables. Por ejemplo, los profesionales dentro de la sanidad y del sistema judicial necesitan estar familiarizados con formas de expresión estándares y coloquiales, empleadas con regularidad en la consulta del doctor o dentro del sistema judicial, para poder comunicarse satisfactoriamente con sus pacientes o clientes. Un estudiante con un repertorio lingüístico amplio podrá decidir qué variedades usar, por qué, cuándo y con quién. En contraste, una persona competente únicamente en la variedad estándar (como suele ser el caso de los EESL) estará en gran desventaja a la hora de comunicarse con hablantes de variedades del español de EE. UU. más coloquiales.

El conocimiento sociolingüístico es primordial para alcanzar este objetivo. Leeman (2005, 2012) y Martínez (2003), entre otros, enfatizan la necesidad de que tanto los profesores como los estudiantes comprendan y estén conscientes de la variación lingüística y la complejidad de las políticas e ideologías lingüísticas. Los autores sostienen que es fundamental incorporar una amplia gama de conceptos sociolingüísticos en el currículum del español como LH (p. ej., variación lingüística; multialfabetización; y conocimiento de los aspectos sociales, culturales y políticos de la lengua). El conocimiento sociolingüístico ayudará a los estudiantes a desarrollar una concientización lingüística crítica (Fairclough, 1995) la cual les permitirá a su vez examinar críticamente el papel de las ideologías lingüísticas y de las jerarquías políticas y sociales en relación a las lenguas para comprender mejor su propia situación lingüística.

Suponiendo que el currículum de las clases de LH está enmarcado dentro de un enfoque sociolingüístico que promueve una concientización lingüística y el pensamiento crítico, las técnicas contrastivas que fomentan un discernimiento de las diferencias entre las variedades lingüísticas son esenciales para alcanzar resultados positivos con los estudiantes (Fairclough, 2005). Los EELH necesitan identificar las formas para ser conscientes de las diferencias entre las muchas variedades de la LH y así poder decidir qué formas usar y en qué contextos (the noticing hypothesis: Schmidt, 1990, 2001, 2010). La yuxtaposición de la misma expresión en dos variedades lingüísticas ayuda a que los estudiantes adviertan las diferencias entre ellas, lo que facilita a su vez el proceso de adquisición (James, 1996). La instrucción debería centrarse particularmente en subrayar diferencias sutiles, ya que los estudiantes pueden reconocer fácilmente las más obvias. Una vez que los estudiantes 
identifican las diferentes formas, también son capaces de diferenciarlas más fácilmente entre sí y adquieren la habilidad de emplearlas conscientemente (Fairclough, s.f.). Por ejemplo, al enseñarles el término general 'impuestos' como una alternativa al préstamo generalizado 'taxas', los estudiantes pueden percibir la diferencia entre las dos formas fácilmente; sin embargo, el siguiente ejemplo tomado del estudio de Fairclough (2005) sirve para ilustrar que hay ciertas diferencias sutiles entre las variedades que no son identificadas tan fácilmente por los estudiantes:

\section{1.a Yo podía ser mejor estudiante si tratara. \\ 1.b Yo podría ser mejor estudiante si tratara.}

En el ejemplo (1.a), el estudiante produjo la forma de imperfecto de indicativo podía, mientras que la forma esperada sería el condicional podría (1.b). Seguramente, dada la mínima distancia lingüística entre las dos formas, el estudiante no se dio cuenta de que son dos formas diferentes.

Una estrategia para lograr la expansión del repertorio lingüístico de los EELH es mediante la multialfabetización, o sea, el uso de una multiplicidad de discursos de tipo multimodal con actividades interpretativas y productivas, frecuentemente realizadas de modo colaborativo, siempre enmarcadas en un enfoque crítico (López-Sánchez, 2014, p. 289). Samaniego y Warner (s.f.) señalan que el término, acuñado por primera vez por el New London Group (NLG, 1996), "reconoce la variabilidad y la contingencia de la comunicación en las sociedades globalizadas contemporáneas, caracterizadas por el uso de nuevas tecnologías y la producción de textos multimodales, así como nociones cambiantes de comunidades discursivas y prácticas literarias" (mi traducción). En otras palabras, equivale a preparar al estudiantado a adaptarse a la continua evolución de la alfabetización utilizando los medios sociales, la literatura, el arte, la publicidad, entre muchos otros.

La multialfabetización permite que los estudiantes desarrollen una competencia translingüística y transcultural (p. ej., Schechtman y Koser, 2008; Wellmon, 2008). Dentro del bilingüismo, el translenguar se entiende como el uso simultáneo de varias lenguas para comunicarse. El concepto de translenguar se opone a la idea de que un bilingüe es como dos monolingües en uno, afirmación que ya ha sido cuestionada por Grosjean (1985), Cook (1997) y más recientemente por Valdés (2006), entre muchos otros. García (2013, p. 354) define el término "translenguar" como el "conjunto de prácticas discursivas complejas de todos los bilingües y las estrategias pedagógicas que utilizan esas prácticas discursivas para liberar las maneras de hablar, ser y conocer de comunidades bilingües subalternas". Según la autora, este fenómeno no se limita al cambio de códigos y pedagógicamente puede fomentarse a través de actividades de traducción, escritura, diálogos, lecturas, etc.

Dentro del campo de la enseñanza de lenguas, muchos expertos han argumentado a favor del uso de material auténtico y de crear, siempre que sea posible, contextos lingüísticos reales que se aproximen a los intercambios comunicativos de la vida real. Una de las mejores maneras de hacer que la enseñanza de lenguas sea 'real' es crear conexiones con la comunidad de la lengua nativa. Preferiblemente, dichas conexiones deberían surgir primero dentro de un contexto familiar (familia y amigos), en los estudiantes que tienen niveles bajos de competencia lingüística, e ir ampliándose paulatinamente a la comunidad en un sentido más amplio y al mundo a medida que evolucionen sus habilidades lingüísticas en las variedades de la lengua de herencia (Fairclough y Belpoliti, 2013). En el campo de la enseñanza de LE, cada 
vez más instituciones de educación superior parecen estar siguiendo este modelo en expansión mediante (a) el diseño de proyectos culturales dentro del currículum; (b) la incorporación de la metodología aprendizaje-servicio en la instrucción; y (c) la oferta de programas de estudio en países hispanohablantes para EELH.

En cuanto al diseño e implementación de proyectos culturales dentro del currículum de LE, Fairclough y Belpoliti (2013) proponen una serie de proyectos culturales, basados en actividades de andamiaje y de investigación, diseñados para desarrollar la competencia lingüística de estudiantes de herencia de diferentes niveles. Los EELH recogen datos mediante varias herramientas (encuestas, Internet, entrevistas, etc.) para llevar a cabo proyectos de investigación que se extienden de un contexto inmediato (familia y amigos) a la comunidad (compañeros y luego la comunidad en general) con el objeto de familiarizarse más con los distintos aspectos de la cultura de la LE. Los estudiantes desarrollan el proyecto a lo largo del semestre, siguiendo una metodología de andamiaje y con el apoyo constante del instructor. Al final del semestre, presentan sus resultados mediante ensayos escritos y presentaciones orales que requieren niveles crecientes de competencia lingüística según la secuenciación del curso de español como LH. Para estos proyectos, los estudiantes suelen usar su propia variedad lingüística durante las entrevistas o procesos de recolección de datos, mientras que se espera que empleen formas estándares en sus trabajos finales escritos y orales. En ocasiones, los trabajos finales suelen exhibir una mezcla de ambos dialectos si los estudiantes citan directamente de las entrevistas.

Una vez que los estudiantes están listos para dejar a un lado los contextos familiares, como los descritos en el párrafo anterior, es esencial vincular a los EELH con diferentes profesiones donde puedan hacer uso de sus destrezas lingüísticas (Martínez, 2010). Los cursos como los de español para fines específicos (Martínez y Schwartz, 2012), las pasantías o trabajos voluntarios (Community Service Learning; Martínez, s.f.), los estudios en otros países de habla hispana (Riegelhaupt y Carrasco, 2000), todos ellos junto con las prácticas innovadoras presentadas anteriormente conducen a forjar una conexión más estrecha entre el EELH y la comunidad de habla, que a su vez conlleva al mantenimiento de la lengua minoritaria en una situación de contacto lingüístico.

\section{Conclusiones}

En Estados Unidos, la profesionalización de la enseñanza del español como LH es la prioridad número uno dentro del campo. En la actualidad, existe una carencia de programas exhaustivos destinados a entrenar profesionales en esta disciplina. La falta de entrenamiento de los instructores es aún más evidente en los programas de estudio en el extranjero. Muchos de los profesionales que imparten cursos de español cuentan con entrenamiento y experiencia en español como segunda lengua, pero no están familiarizados con el campo de la enseñanza de lenguas de herencia. Es probable que carezcan de conocimiento sociolinguístico, que desconozcan las necesidades socio-afectivas de los estudiantes de herencia y que no cuenten con el entrenamiento necesario para poner en práctica técnicas que potencien la competencia linguiística de los EELH.

Para poder implementar las pedagogías innovadoras someramente presentadas en este artículo es necesario contar con profesionales que conozcan el perfil y las necesidades lingüísticas del estudiantado de español como LH, que estén al tanto de las investigaciones más 
recientes en cuanto a la enseñanza de las LH, y que estén dispuestos a adaptar sus programas y métodos de enseñanza basando sus decisiones en las sugerencias pedagógicas de dichas investigaciones.

Por medio del reconocimiento y validación de la variación lingüística desde una perspectiva crítica expresada por medio del conocimiento sociolingüístico, siguiendo un modelo multidialectal que puede ponerse en práctica siguiendo ciertos principios que guían la adquisición de un segundo dialecto, el translenguar y la multialfabetización que permiten que los EELH tengan la posibilidad de tomar sus propias decisiones lingüísticas, se pueden establecer verdaderas conexiones con la comunidad de habla, las cuales se van expandiendo paulatinamente de la comunidad local hacia la global y conducen a un compromiso social cuyo objetivo primordial es promover el mantenimiento de la lengua de herencia en situaciones de contacto lingüístico.

\section{Bibliografía}

Au, T., Knightly, L., Jun, S. A., y Oh, J. (2002). Overhearing a language during childhood. Psychological Science. 13, 238-243.

Beaudrie, S. M. (2009). Spanish receptive bilinguals: Understanding the cultural and linguistic profile of learners from three different generations. Spanish in Context. 6 (1), 85-104.

Beaudrie, S. M. (2012). Research on University-Based Spanish Heritage Language Programs in the United States: The Current State of Affairs. Por S. M. Beaudrie y M. Fairclough (Eds.). Spanish as a Heritage Language in the United States: The State of the Field. (203-222). Washington, D.C.: Georgetown University Press.

Beaudrie, S. M. (2015). Approaches to Language Variation: Goals and Objectives of the Spanish Heritage Language Syllabus. Heritage Language Journal. 12 (1), 1-21.

Bernal-Enríquez, Y. y Hernández-Chávez, E. (2003). La enseñanza del español en Nuevo México: ¿revitalización o erradicación de la variedad chicana? Por A. Roca and M. C. Colombi (Eds.). Mi lengua: Spanish as a heritage language in the United States. Washington, DC: Georgetown University Press.

Bowles, M. (2011). Exploring the role of modality: Second language-heritage learner interactions in the Spanish language classroom. Heritage Language Journal. 8 (1), 30-65.

Carreira, M. (2012). The Advanced Speaker: An Overview of the Issues in Heritage Language Teaching. [pdf.]. http://international.ucla.edu/media/files/CarreiraPositionPaperFinal. pdf [Consulta 8 de enero de 2015].

Carvalho, A. (2012). Code-switching: From Theoretical to Pedagogical Considerations. Por S. M. Beaudrie y M. Fairclough (Eds.). Spanish as a Heritage Language in the United States: The State of the Field. (139-157). Washington, DC: Georgetown University Press.

Cook, V. (1997). The consequences of bilingualism and cognitive processing. Por A. M. B. de Groot y J. F. Kroll (Eds.). Tutorials in Bilingualism: Psycholinguistic Perspectives. (279-299). Mahwah: Lawrence Erlbaum. 
Correa, M. (2011). Heritage Language Learners of Spanish: What Role does Metalinguistic Knowledge Play in their Acquisition of the Subjunctive? Por L. Ortiz-López (Ed.). Selected Proceedings of the 13th Hispanic Linguistics Symposium. (128-138). Somerville, MA: Cascadilla Proceedings Project.

Fairclough, M. (2003). El (denominado) ‘Spanglish’ en Estados Unidos: polémicas y realidades. Revista Internacional Lingüística Iberoamericana. 2, 185-204.

Fairclough, M. (2005). Spanish and Heritage Language Education in the United States: Struggling with Hypotheticals. Madrid / Frankfurt: Iberoamericana Libros-Vervuert.

Fairclough, M. (s.f.). Incorporating additional varieties to the linguistic repertoires of HL learners: A multidialectal model. Por M. Fairclough y S. M. Beaudrie (Eds). Innovative Approaches in Heritage Language Teaching: From Research to Practice. Washington D.C.: Georgetown University Press.

Fairclough, M. (2011). Testing the Lexical Recognition Task with Spanish/English Bilinguals in the United States. Language Testing. 28 (2), 273-297.

Fairclough, M. y Belpoliti, F. (2013). Houston's Hispanic Community: Understanding Culture through Research in the SHL Classroom. 24 Conference on Spanish in the United States y 9 th Conference on Spanish in Contact with Other Languages. McAllen, TX: UTPA.

Fairclough, N. (1995). Critical Discourse Analysis. Boston: Addison Wesley.

Fishman, J. (2001). 300-plus Years of Heritage Language Education in the United States. Por J. Peyton, D. Ranard, y S. McGinnis (Eds.). Heritage Languages in America: Preserving a National Resource. (81-98). Washington, DC y McHenry, IL: Center for Applied Linguistics y Delta Systems.

Freire, P. (1970). Pedagogy of the Oppressed. New York: Herder y Herder.

García, O. (2013). El papel del translenguar en la enseñanza del español en los Estados Unidos. Por D. Dumitrescu (Ed.). El español en Estados Unidos: e pruribus unum?: Enfoques multidisciplinarios (353-373). Madrid: Santillana.

Grosjean, F. (1985). The Bilingual as a Competent but Specific Speaker-Hearer. Journal of Multilingual and Multicultural Development. 6 (6), 467-477.

Gutiérrez, M. J. y Fairclough, M. (2006). Incorporating Linguistic Variation into the Classroom. Por R. Salaberry y B. Lafford (Eds.). The Art of Teaching Spanish: Second Language Acquisition, from Research to Praxis. (173-192). Washington, D.C.: Georgetown University Press.

Hidalgo, M. (1990). The emergence of standard Spanish in the American continent: Implications for Latin American dialectology. Language Problems and Language Planning. 14 (1), 47-63.

Hidalgo, M. (1997). Criterios normativos e ideología lingüística: aceptación y rechazo del español de los Estados Unidos. Por M. C. Colombi y F. X. Alarcón (Eds.). La enseñanza del español a hispanohablantes: Praxis y teoría. (109-121). Boston, MA: Houghton Mifflin. 
Hislope, K. (2005). A present subjunctive focus-on-form study of heritage speakers of Spanish. Por L. Ortiz-López y M. Lacorte (Eds.). Contactos y contextos lingüísticos: El español en los Estados Unidos y en contacto con otras lenguas. (315-322). Madrid/Frankfurt: Iberoamericana/Vervuert.

James, C. (1996). Mother Tongue Use in Bilingual/Bidialectal Education: Implications for Bruneian Dwibahasa. Journal of Multilingual and Multicultural Development. 17, 248-57.

Jo, H-Y. (2001). 'Heritage' Language Learning and Ethnic Identity: Korean Americans'Struggle with Language Authorities. Language, Culture, and Curriculum. 14, $26-41$.

Kondo-Brown, K. (2010). Curriculum Development for Advancing Heritage Language Competence: Recent Research, Current Practices, and a Future Agenda. Annual Review of Applied Linguistics. 30, 24-41.

Leeman, J. (2005). Engaging critical pedagogy: Spanish for native speakers. Foreign Language Annals. 38, 35-45.

Leeman, J. (2012). Investigating Language Ideologies in Spanish as a Heritage Language. Por S. M. Beaudrie y M. Fairclough (Eds.). Spanish as a Heritage Language in the United States: The State of the Field. (43-60). Washington, D.C.: Georgetown University Press.

Leeman, J. (2014). Critical approaches to the teaching of Spanish as a local-foreign language. Por M. Lacorte (Ed.). The Handbook of Hispanic Applied Linguistics. (275-292). New York/London: Routledge.

Lipski, J. (1993). Creoloid phenomena in the Spanish of transitional bilinguals. Por A. Roca y J. Lipski (Eds.). Spanish in the United States: Linguistic contact and diversity. (155-182). Berlin: Mouton de Gruyter.

López-Sánchez, A. (2014). Hacia una pedagogía para la multialfabetización: El diseño de una unidad didáctica inspirada en las propuestas del New London Group. Hispania. 97 (2), 281-297.

Martínez, G. (2003). Classroom based dialect awareness in heritage language instruction: A critical applied linguistic approach. Heritage Language Journal. 1 (1), 44-57.

Martínez, G. (2010). Medical Spanish for Heritage Learners: A Prescription to Improve the Health of Spanish-Speaking Communities. Por S. Rivera-Mills y J. A. Trujillo (Eds.). Building Communities and Making Connections. (2-15). Newcastle Upon Tyne: Cambridge Scholars Publishing.

Martínez, G. (s.f.). Goals and beyond in HL Education. Por M. Fairclough y S. M. Beaudrie (Eds.). Innovative Approaches in Heritage Language Teaching: From Research to Practice. Washington D.C.: Georgetown University Press.

Martínez, G. y Schwartz, A. (2012). Elevating "low" language for high stakes: A case for critical community-based learning in a Medical Spanish for heritage learners program. Heritage Language Journal. 9 (2), 175-186.

Mikulski, A. M. (2010). Receptive volitional subjunctive abilities in heritage and traditional foreign language learners of Spanish. Modern Language Journal. 94 (2), 217-233. 
Montrul, S. (2011). Morphological errors in Spanish second language learners and heritage speakers. Studies in Second Language Acquisition. 33 (2), 163-192.

Montrul, S., y Bowles, M. (2010). Is grammar instruction beneficial for heritage language learners: Dative case marking in Spanish. Heritage Language Journal. 7 (1), $47-73$.

Montrul, S., Foote, R., y Perpiñán, S. (2008). Gender agreement in adult second language learners and Spanish heritage speakers: The effects of age and context of acquisition. Language Learning. 58 (3), 503-553.

NLG, New London Group. (1996). A Pedagogy of Multiliteracies: Designing social futures. Harvard Educational Review. 66 (1). http://vassarliteracy.pbworks.com/f/ Pedagogy+of+Multiliteracies_New+London+Group.pdf [Consulta 12 de marzo de 2015].

Oh, J., y Au, T. K-F. (2005). Learning Spanish as a heritage language: The role of sociocultural background variables. Language, Culture, and Curriculum. 18 (3), 229-241.

Parodi, C. (2008). Stigmatized Spanish inside the classroom and out: a model language teaching to heritage speakers. Por D. M. Brinton y O. Kagan (Eds.). Heritage Language Acquisition: A New Field Emerging. (199-214). Mahwah, NJ: Lawrence Erlbaum.

Parra, M. L. (2013). Expanding Language and Cultural Competence in Advanced Heritageand Foreign- Language Learners through Community Engagement and Work with the Arts. Heritage Language Journal. 10 (2). http://www.heritagelanguages.org/ [Consulta 8 de febrero de 2015].

Pew Research Center. 5 facts about Hispanics for Hispanic Heritage Month. http://www. pewresearch.org/fact-tank/2013/09/17/5-facts-about-hispanics/ [Consulta 7 de febrero de 2015].

Potowski, K., Jegerski, J., y Morgan-Short, K. (2009). The effects of instruction on linguistic development in Spanish heritage language speakers. Language Learning. 59, 537-579.

Ryan, C. (2013). Language Use in the United States. American Community Language Survey Reports. [pdf.]. http://www.census.gov/prod/2013pubs/acs-22.pdf [Consulta 7 de febrero de 2015].

Riegelhaupt, F. y Carrasco, R. L. (2000). Mexico host family reactions to a bilingual Chicana teacher in Mexico: A case study of language y culture clash. Bilingual Research Journal. 24, 405-421.

Rouchdy, A. (2002). Language Conflict and Identity: Arabic in the American Diaspora. Por A. Rouchdy (Ed.). Language Contact and Language Conflict in Arabic: Variations on a Sociolinguistic Theme. (133-48). London: Routledge/Curzon.

Samaniego, M. y Warner, Ch. (s.f.). A 'Multiliteracies' approach to heritage language instruction. Por M. Fairclough y S. M. Beaudrie (Eds.). Innovative Approaches in Heritage Language Teaching: From Research to Practice. Washington D.C.: Georgetown University Press.

Schechtman, R. R., y Koser, J. (2008). Foreign Languages and Higher Education: A Pragmatic Approach to Change. The Modern Language Journal. 92 (2), 309-312.

Schmidt, R. (1990). The Role of Consciousness in Second Language Learning. Applied Linguistics. 11, 129-58. 
Schmidt, R. (2001). Attention. Por P. Robinson (Ed.). Cognition and Second Language Instruction. (3- 32). Cambridge: Cambridge University Press.

Schmidt, R. (2010). Attention, Awareness, and Individual Differences in Language Learning. Por W. M. Chan, S. Chi, K. N. Cin, J. Istanto, M. Nagami, J. W. Sew, T. Suthiwan y I. Walker. Proceedings of CLaSIC 2010, Singapore, December 2-4. (721-37). Singapore: National University of Singapore, Centre for Language Studies.

Silva-Corvalán, C. (1994). Language Contact and Change: Spanish in Los Angeles. Oxford: Oxford University Press.

United States Census Bureau. (2012). The Hispanic Population in the United States: 2012. http://www.census.gov/population/hispanic/data/2012.html [Consulta 7 de febrero de 2015].

Valdés, G. (1978). A comprehensive approach to the teaching of Spanish to bilingual Spanishspeaking students. Modern Language Journal. 63 (3), 101-110.

Valdés, G. (1997). The teaching of Spanish to heritage Spanish-speaking students: Outstanding issues y unanswered questions. Por M. C. Colombi y F. X. Alarcón (Eds.). La ensenañza del español a hispanohablantes: Praxis y teoría. (8-44). Boston, MA: Houghton Mifflin.

Valdés, G. (2005). Bilingualism, heritage language learners, y SLA research: Opportunities lost or seized? Modern Language Journal. 89 (3), 410-426.

Valdés, G. (2006). The Spanish Language in California. Por G. Valdés, J. Fishman, R. Chávez y W. Pérez (Eds.). Developing Minority Language Resources: The Case of Spanish in California (24-53). Clevedon: Multilingual Matters.

Valdés, G. y Geoffrion-Vinci, M. (1998). Chicano Spanish: The Problem of the 'Underdeveloped' Code in Bilingual Repertoires. The Modern Language Journal. 82 (4), 473-501.

Veltman, C. (1983). Language Shift in the United States. Berlin: Mouton.

Veltman, C. (2000). The American linguistic mosaic: understanding language shift in the United States. Por S. L. Mckay y S. C. Wong (Eds.). New Immigrants in the United States. (58-93). Cambridge: Cambridge University Press.

Wellmon, C. (2008). Languages, Cultural Studies, and the Futures of Foreign Language Education. The Modern Language Journal. 92 (2), 292-95.

Zentella, A. C. (1997). Growing up Bilingual: Puerto Rican Children in New York City. Oxford: Blackwell. 
